\title{
An Efficient Synthesis of 3,4-Dihydro-3-substituted-2H-naphtho[2,1-e][1,3]oxazine Derivatives Catalyzed by Zirconyl(IV) Chloride and Evaluation of its Biological Activities
}

\author{
Amol H. Kategaonkar, Swapnil S. Sonar, Rajkumar U. Pokalwar, Atul H. Kategaonkar, ${ }^{\dagger}$ Bapurao B. Shingate, \\ and Murlidhar S. Shingare* \\ Department of Chemistry, Dr. Babasaheb Ambedkar Marathwada University, Aurangabad 431004, Maharashtra, India \\ ${ }^{*}$ E-mail:prof_msshingare@rediffmail.com \\ †Pharmacology Laboratory, Maharashtra Institute of Pharmacy, Pune 411 038, Maharashtra, India \\ Received February 20, 2010, Accepted April 2, 2010
}

\begin{abstract}
An efficient and novel one-pot synthesis of new 3,4-dihydro-3-substituted-2H-naphtho[2,1-e][1,3]oxazine derivatives from 1-naphthol, various anilines and formalin at room temperature grinding is presented. The six-membered $\mathrm{N}, \mathrm{O}$-heterocyclic skeleton was constructed via zirconyl(IV) chloride promoted Mannich type reaction. In vitro antimicrobial activities of synthesized compounds have been investigated against Gram-positive Bacillus subtilis, Gram negative Escherichia coli and two fungi Candida albicans and Aspergillus niger in comparison with standard drugs. The results of preliminary bioassay indicate that some of title compounds possess significant antibacterial and antifungal activity.
\end{abstract}

Key Words: 1,3-Oxazines, Zirconyl(IV) chloride, Grinding, Antibacterial, Antifungal

\section{Introduction}

The Mannich reaction has been widely used ${ }^{1,2}$ to introduce oxazines into a variety of organic compounds. The Mannich reaction involving phenols, formalin and primary amines has been used as a convenient source for a variety of compounds. The interest on 1,3-oxazine molecules has recently increased, mainly due to compounds containing dihydro-1,3-oxazine ring system exhibited a wide spectrum of pharmacological activities such as anti-tumor, ${ }^{3}$ anti-bacterial, ${ }^{4}$ anti-HIV ${ }^{5}$ and antimalarial agents ${ }^{6}$ and their versatility as synthetic intermediates. ${ }^{7}$ This has been the prime driving force for the synthesis of various compounds incorporating the 1,3-oxazine moiety. In addition, naphthoxazine derivatives have exhibited therapeutic potential for the treatment of Parkinson's disease. ${ }^{8,9}$

Multicomponent reactions (MCRs), defined as one pot reactions in which at least three functional groups join through covalent bonds, have been steadily gaining importance in synthetic organic chemistry. ${ }^{10-13}$ The reagents employed may be different molecules or they may be different functional groups of the same reagent. Speed, diversity, efficiency and environmental amiability are some of the key features of this class of reactions.

Recently, $\mathrm{ZrOCl}_{2}$ chemistry ${ }^{14-19}$ has attracted much attention as zirconyl(IV) chloride has emerged as an alternative safe, economical, air and moisture tolerant Lewis acid which has been used in various organic transformations. Several methods for the preparation of 1,3-oxazine derivatives have previously been reported. ${ }^{20-24}$

As per our interest ${ }^{25-30}$ to develop better protocols for the synthesis of biologically active heterocyclic molecules, we would like to report the synthesis of a series of new 3,4-dihydro-3substituted-2H-naphtho[2,1-e][1,3]oxazine derivatives using 1 -naphthol, formalin and various anilines as substrates in pre- sence of zirconyl(IV) chloride $\left(\mathrm{ZrOCl}_{2}\right)$ catalyst. To the best of our knowledge there is no report on the one-pot synthesis of 3,4-dihydro-3-substituted-2H-naphtho[2,1-e][1,3] oxazines using $\mathrm{ZrOCl}_{2}$ as a catalyst.

\section{Results and Discussion}

Chemistry. Initially, a one-pot, three component reaction of 1-naphthol, aniline and formalin (1:1:2) was planned as a model reaction (Scheme 1). The effect of various Lewis acids such as $\mathrm{AlCl}_{3}, \mathrm{SnCl}_{4}, \mathrm{ZnCl}_{2}, \mathrm{TiCl}_{4}, \mathrm{BF}_{3} \cdot \mathrm{OEt}_{2}$ and $\mathrm{ZrOCl}_{2}$ was tabulated in Table 1. The zirconyl(IV) chloride gave a high yield of 3,4dihydro-3-phenyl-2H-naphtho[2,1-e][1,3]oxazine $4 \mathbf{a}$ within a short time, a high molar percentage of the catalyst was required. However, $\mathrm{ZrOCl}_{2}$ alone was found to be the most effective Lewis acid catalyst for the one-pot, three-component synthesis of 4 in good yields. We also observed in the model reaction with vigorous stirring, the product yields much less (45\%) as compared with grinding method $(80 \%)$, therefore we preferred grinding method.

In order to investigate the concentration of catalyst loading for model reaction, the procedure was optimized using different molar concentrations of $\mathrm{ZrOCl}_{2}$ under solvent free conditions at room temperature using grinding (Table 2). High yield of

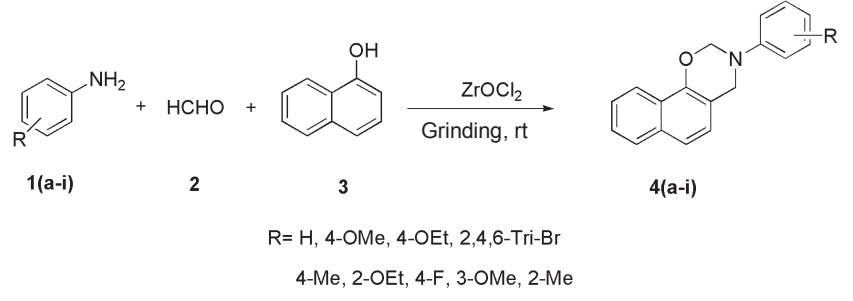

Scheme 1. Reaction of various anilines with formalin and 1-naphthol 
Table 1. Effect of different Lewis acids on synthesis of $\mathbf{4} \mathbf{a}^{a}$

\begin{tabular}{cccc}
\hline Entry & Lewis acid & Time $(\min )$ & ${\text { Yield }(\%)^{b}}^{b}$ \\
\hline 1 & $\mathrm{No} \mathrm{cat.}_{2}$ & 24.0 & - \\
2 & $\mathrm{AlCl}_{3}$ & 12.0 & 25 \\
3 & $\mathrm{ZnCl}_{4}$ & 16.0 & 30 \\
4 & $\mathrm{SnCl}_{4}$ & 8.0 & 37 \\
5 & $\mathrm{BF}_{3} \cdot \mathrm{OEt}_{2}$ & 10.0 & 28 \\
6 & $\mathrm{TiCl}_{4}$ & 18.0 & 16 \\
7 & $\mathrm{ZrOCl}_{2}$ & 0.5 & 80
\end{tabular}

Reaction conditions: ${ }^{a}$ Formalin $(2.0 \mathrm{mmol})$, aniline $(1.0 \mathrm{mmol})$, 1-naphthol $(1.0 \mathrm{mmol})$, catalyst $(15-20 \mathrm{~mol} \%)$, rt, grinding. ${ }^{b}$ Isolated yields.

Table 2. Effect of catalyst loading on the one-pot, three component synthesis of $\mathbf{4 a}^{a}$

\begin{tabular}{cccc}
\hline Entry & $\mathrm{ZrOCl}_{2}(\%)^{b}$ & Time $(\min )$ & Yield $(\%)^{c}$ \\
\hline 1 & 5 & 3.0 & 43 \\
2 & 10 & 1.5 & 48 \\
3 & 15 & 1.0 & 65 \\
4 & 20 & 0.5 & 80 \\
5 & 25 & 0.5 & 80
\end{tabular}

Reaction conditions: ${ }^{a}$ Formalin $(2.0 \mathrm{mmol})$, aniline $(1.0 \mathrm{mmol}), 1$-naphthol $(1.0 \mathrm{mmol})$, rt, grinding. ${ }^{b}$ Zirconyl(IV) chloride ( $\left.\mathrm{mol} \%\right)$. ${ }^{c}$ Isolated yields.

product $4 \mathbf{a}$ was observed using $20 \mathrm{~mol} \%$ of catalyst, while with $5-15 \mathrm{~mol} \%$ of catalyst yields were not good. From these results, it was evident that, the concentration of catalyst plays a crucial role to improve the result to greater extent. It was also observed that, $25 \mathrm{~mol} \%$ of catalyst has no greater change in yields of product. The $\mathrm{ZrOCl}_{2}$ can be used repeatedly for several times without appreciable loss in activity.

To explore, the generality and scope of this method, a wide variety of substituted aromatic anilines were reacted with formalin and 1-naphthol under the similar experimental conditions to afford the corresponding 3,4-dihydro-3-substituted-2Hnaphtho[2,1-e][1,3] oxazine derivatives. $\mathrm{ZrOCl}_{2}$ was found to be compatible with various substituents (electron withdrawing as well as donating substituents) such as $\mathrm{OMe}, \mathrm{OEt}, \mathrm{Me}, \mathrm{F}$ and $\mathrm{Br}$. The resultant products were obtained in good to excellent yields in short reaction times (Table 3 ). The formations of products were characterized by physical and spectroscopic data. The ${ }^{1} \mathrm{H}$ NMR spectra of compounds (4a-i) showed typical two singlet peaks in the region $4.87-4.90 \mathrm{ppm}$ and $5.4-5.7 \mathrm{ppm}$, which are due to protons of $-\mathrm{Ar}-\mathrm{CH}_{2}-\mathrm{N}-$ and $-\mathrm{O}-\mathrm{CH}_{2}-\mathrm{N}$ - of oxazine ring.

Biological results. In vitro antibacterial and antifungal activity was screened by considering zone of inhibition of growth. The synthesized compounds (4a-i) were screened with their different concentrations against $B$. subtilis, E. coli, C. albicans and $A$. niger (Table 4). In vitro antibacterial and antifungal activity of our designed compounds shows moderate MIC values in between $10-25 \mu \mathrm{g} / \mathrm{mL}$ against $B$. subtilis, E. coli, C. albicans and $A$. niger in vitro as shown in (Table 4).

The investigation of antibacterial screening data revealed that, all the tested compounds showed considerable and varied activity against the two human pathogenic bacteria. Compounds
Table 3. Reaction of various anilines with 1-naphthol and formalin $(1: 1: 2)$

\begin{tabular}{cccccc}
\hline Entry & Compound & $\mathrm{R}$ & $\begin{array}{c}\text { Time } \\
(\mathrm{min})\end{array}$ & $\begin{array}{c}\text { Yield } \\
(\%)^{a}\end{array}$ & $\begin{array}{c}\mathrm{mp} \\
\left({ }^{\circ} \mathrm{C}\right)\end{array}$ \\
\hline 1 & $\mathbf{4 a}$ & $\mathrm{H}$ & 0.5 & 80 & $62-63$ \\
2 & $\mathbf{4 b}$ & $4-\mathrm{OMe}$ & 1.0 & 74 & $300(\mathrm{~d})$ \\
3 & $\mathbf{4 c}$ & $4-\mathrm{OEt}$ & 0.5 & 72 & $76-77$ \\
4 & $\mathbf{4 d}$ & $2,4,6-\mathrm{Tri} \mathrm{Br}$ & 0.5 & 73 & $72-74$ \\
5 & $\mathbf{4 e}$ & $4-\mathrm{Me}$ & 1.0 & 81 & $196-198$ \\
6 & $\mathbf{4 f}$ & $2-\mathrm{OEt}$ & 0.5 & 89 & $200(\mathrm{~d})$ \\
7 & $\mathbf{4 g}$ & $4-\mathrm{F}$ & 0.5 & 83 & $118-120$ \\
8 & $\mathbf{4 h}$ & 3-OMe & 0.5 & 87 & $280(\mathrm{~d})$ \\
9 & $\mathbf{4 i}$ & $2-\mathrm{Me}$ & 0.5 & 78 & $86-88$ \\
\hline
\end{tabular}

${ }^{a}$ Isolated yield.

Table 4. Antibacterial and antifungal activity of compounds (4a-i)

\begin{tabular}{ccccc}
\hline \multirow{2}{*}{ Compound } & $\begin{array}{c}\text { Bacillus } \\
\text { subtilis }\end{array}$ & $\begin{array}{c}\text { Escherichia } \\
\text { coli }\end{array}$ & $\begin{array}{c}\text { Candida } \\
\text { albicans }\end{array}$ & $\begin{array}{c}\text { Aspergillus } \\
\text { niger }\end{array}$ \\
\cline { 2 - 5 } & $\mathrm{ZI}^{a}(\mathrm{MIC})^{b}$ & $\mathrm{ZI}(\mathrm{MIC})$ & $\mathrm{ZI}(\mathrm{MIC})$ & $\mathrm{ZI}(\mathrm{MIC})$ \\
\hline $\mathbf{4 a}$ & $14.0(25)$ & $13.4(15)$ & $12.4(25)$ & $14.1(15)$ \\
$\mathbf{4 b}$ & $16.0(10)$ & $13.6(25)$ & $13.0(20)$ & $12.4(25)$ \\
$\mathbf{4 c}$ & $\mathbf{1 8 . 0 ( 1 0 )}$ & $12.0(15)$ & $\mathbf{1 8 . 2 ( 1 0 )}$ & $14.0(15)$ \\
$\mathbf{4 d}$ & $\mathbf{1 8 . 0 ( 1 0 )}$ & $14.0(15)$ & $12.2(15)$ & $13.2(20)$ \\
$\mathbf{4 e}$ & $15.0(20)$ & $13.2(15)$ & $14.2(20)$ & $11.1(25)$ \\
$\mathbf{4 f}$ & $14.0(15)$ & $12.0(20)$ & $15.6(15)$ & $14.2(15)$ \\
$\mathbf{4 g}$ & $\mathbf{1 8 . 0 ( 1 0 )}$ & $13.2(15)$ & $14.0(15)$ & $15.4(10)$ \\
$\mathbf{4 h}$ & $12.2(15)$ & $13.4(25)$ & $16.0(10)$ & $\mathbf{1 6 . 1 ( 1 0 )}$ \\
$\mathbf{4 i}$ & $14.4(20)$ & $13.0(15)$ & $13.8(15)$ & $12.1(25)$ \\
Strept. & $17.6(10)$ & $17.3(10)$ & n.t. ${ }^{c}$ & n. t. \\
Gris. & n.t. & n.t. & $17.2(10)$ & $16.9(10)$ \\
\hline
\end{tabular}

${ }^{a}$ Zone of inhibition in $\mathrm{mm},{ }^{b}$ minimum inhibitory concentration in $\mu \mathrm{g} / \mathrm{mL}$, cn. t. not tested.

4c ( $\mathrm{R}=4$-OMe), $4 \mathbf{d}(\mathrm{R}=2,4,6$-tri Bromo) and $\mathbf{4 g}(\mathrm{R}=4-\mathrm{F})$ showed the zone of inhibition $(18.0 \mathrm{~mm}$ each $)$ as that of the standard streptomycin $(17.6 \mathrm{~mm})$ against $B$. subtilis. Against E. coli compounds have not shown significant activity. The present data indicate a little change in the in the substituent might also affect the antibacterial activity of title compounds (4a-i). Comparison of biological activities among (4a-i) shows functional groups as $\mathrm{R}=\mathrm{OMe} / \mathrm{Br} / \mathrm{F}$ to be potentially more active against $B$. subtilis. Also, (Table 4) clearly reveals that, compounds $4 \mathbf{c}, \mathbf{4 d}$ and $\mathbf{4 g}$ are more effective than the standard streptomycin.

The antifungal activity of compounds (4a-i) (Table 4) clearly indicates that, compound $4 \mathbf{c}(\mathrm{R}=4-\mathrm{OEt})$ exhibited $(18.2 \mathrm{~mm})$ zone of inhibition which is more than that of standard Griseofluvin against $C$. albicans. Against $A$. niger compound $\mathbf{4 h}$ (3$\mathrm{OMe})$ showed $(16.1 \mathrm{~mm})$ zone of inhibition as that of standard Griseofluvin (16.8 mm).

\section{Conclusions}

In conclusion, the salient features of this method include high yields, short reaction times, cleaner reaction profile and the 
use of inexpensive and readily available $\mathrm{ZrOCl}_{2}$ as a catalyst. Also the antimicrobial activities of all the newly synthesized compounds have been evaluated. The antibacterial and antifungal activities were observed in all the tested compounds. Most of the compounds prove even better than the standard drugs. The importance of such work lies in the possibility that the new compounds might be more efficacious drugs against bacteria and fungi, which could be helpful in designing more potent antibacterial and antifungal agents for therapeutic use.

\section{Experimental Section}

All chemicals and solvents were purchased from Merck (Darmstadt, Germany), Spectrochem (Mumbai, India) and S. D. Fine-chem. (Mumbai, India). Melting points were determined in open capillaries on Kumar's melting point apparatus (India) and are uncorrected. IR spectra were recorded on JASCO FT-IR 4100, Japan using KBr discs. ${ }^{1} \mathrm{H}$ NMR spectra were recorded on Varian NMR spectrometer, Model Mercury Plus (400 MHz), ${ }^{13} \mathrm{C}$ NMR Spectra were recorded on the Bruker DRK300. Mass spectra [ES-MS] were recorded on a Water-Micro mass Quattro-II spectrophotometer. Elemental analyses were performed on CHNS analyzer Flash 1112, Thermo Finnigan. The progress of the reactions was monitored by TLC on Merck silica plates. Results are presented as, chemical shift $\delta$ in ppm. Multiplicities are shown as the abbreviations: $\mathrm{s}$ (singlet), $\mathrm{t}$ (triplet), q (quartet), m (multiplet). Solvents were commercially available materials of reagent grade.

General procedure for synthesis of 3,4-dihydro-3-substituted-2H-naphtho[2,1-e][1,3]oxazine derivatives. A mixture of formalin $(20 \mathrm{mmol})$, aromatic amine $(10 \mathrm{mmol})$, 1-naphthol $(10 \mathrm{mmol})$ and $\mathrm{ZrOCl}_{2}(20 \mathrm{~mol} \%)$ was ground at room temperature. The progress of the reaction was monitored on TLC. After completion of reaction, reaction mixture was extracted with methylene dichloride $(3 \times 50 \mathrm{~mL})$, washed with water $(2 \times$ $10 \mathrm{~mL})$ and brine $(2 \times 20 \mathrm{~mL})$. Thus separated organic layer was dried over anhydrous $\mathrm{Na}_{2} \mathrm{SO}_{4}$. The solvent was removed under reduced pressure. The obtained crude product was purified by column chromatography on silica gel by hexane: ethyl acetate as an eluent.

3,4-Dihydro-3-phenyl-2H-naphtho[2,1-e][1,3] oxazine (4a): IR (KBr, $v_{\max } / \mathrm{cm}^{-1}$ ): 1030 (sym. C-O-C), 1214 (asym. C-O-C); ${ }^{1} \mathrm{H}$ NMR (DMSO- $d_{6}, 400 \mathrm{MHz}, \delta$ ppm): 4.77 (s, 2H, -Ar- $\mathrm{CH}_{2}-\mathrm{N}-$ ), 5.41 (s, 2H, -O-CH $2-\mathrm{N}-), 6.82-7.56(\mathrm{~m}, 11 \mathrm{H}, \mathrm{Ar}-\mathrm{H}) ;{ }^{13} \mathrm{C} \mathrm{NMR}$ (DMSO- $d_{6}, 75 \mathrm{MHz}, \delta$ ppm): 49.1, 79.4, 112.7, 115.3, 117.5, 119.8, 120.7, 124.2, 125.2, 125.5, 126.0, 127.6, 129.2, 132.9, 147.9, 148.8; MS: $m / z 262.2(\mathrm{~m}+1)$; Elemental analysis: $\mathrm{C}_{18}$ $\mathrm{H}_{15} \mathrm{NO}$ Calcd.: C: $82.73 \%$; H: 5.79\%; N: 5.36\%; Found: C: $82.65 \%$, H: $5.83 \%$, N: $5.48 \%$.

3,4-Dihydro-3-(4-methoxyphenyl)-2H-naphtho[2,1-e][1,3] oxazine (4b): IR (KBr, $v_{\max } / \mathrm{cm}^{-1}$ ): 1019 (sym. C-O-C), 1228 (asym. C-O-C); ${ }^{1} \mathrm{H}$ NMR (DMSO- $d_{6}, 400 \mathrm{MHz}, \delta$ ppm): 3.62 (s, 3H, OMe), 4.87 (s, 2H, -Ar- $\mathrm{CH}_{2}-\mathrm{N}-$ ), 5.40 (s, 2H, -O- $\mathrm{CH}_{2}-$ $\mathrm{N}-), 6.77-7.82$ (m, 10H, Ar-H); ${ }^{13} \mathrm{C}$ NMR (DMSO- $d_{6}, 75 \mathrm{MHz}$, $\delta$ ppm): 48.2, 52.2, 80.1, 111.2, 115.6, 117.4, 119.5, 121.1, 124.2, 125.7, 125.9, 126.8, 127.2, 130.1, 132.3, 146.8, 148.6; MS: $m / z 292.2(\mathrm{~m}+1)$; Elemental analysis: $\mathrm{C}_{19} \mathrm{H}_{17} \mathrm{NO}_{2}$ Calcd.: C: $78.33 \%$; H: 5.88\%; N: 4.81\%; Found: C: 78.45\%, H: 5.90\%,
$\mathrm{N}: 4.72 \%$.

3-(4-Ethoxyphenyl)-3,4-dihydro-2H-naphtho[2,1-e][1,3] oxazine (4c): IR (KBr, $v_{\max } / \mathrm{cm}^{-1}$ ): 1028 (sym. C-O-C), 1224 (asym. C-O-C); ${ }^{1} \mathrm{H}$ NMR (DMSO- $\left.d_{6}, 400 \mathrm{MHz}, \delta \mathrm{ppm}\right): 1.20(\mathrm{t}$, $\left.3 \mathrm{H}, J=8 \mathrm{~Hz}, \mathrm{O}-\mathrm{CH}_{2}-\mathrm{CH}_{3}\right), 3.90$ (q, $2 \mathrm{H}, J=8 \mathrm{~Hz}, \mathrm{O}-\mathrm{CH}_{2}-\mathrm{CH}_{3}$ ), 4.90 (s, 2H, - $\left.\mathrm{Ar}-\mathrm{CH}_{2}-\mathrm{N}-\right), 5.40$ (s, 2H, -O-CH $\left.-\mathrm{N}-\right), 6.80-7.80$ (m, 10H, Ar-H); ${ }^{13} \mathrm{C}$ NMR (DMSO- $d_{6}, 75 \mathrm{MHz}, \delta$ ppm): 14.6, 48.4, 65.1, 80.6, 112.6, 115.9, 117.4, 119.7, 120.1, 123.4, 124.8, 125.2, 125.8, 126.5, 127.7, 129.8, 132.0, 147.2; MS: $m / z 306.2(\mathrm{~m}+1)$; Elemental analysis: $\mathrm{C}_{20} \mathrm{H}_{19} \mathrm{NO}_{2}$ Calcd.: $\mathrm{C}$ : 78.66\%; H: $6.27 \%$; N: 4.59\%; Found: C: $78.71 \%$, H: $6.28 \%$, $\mathrm{N}: 4.24 \%$.

3-(2,4,6-Tribromophenyl)-3,4-dihydro-2H-naphtho[2,1-e] [1,3]oxazine (4d): IR ( $\left.\mathrm{KBr}, v_{\max } / \mathrm{cm}^{-1}\right)$ : 1016 (sym. C-O-C), 1224 (asym. C-O-C); ${ }^{1} \mathrm{H}$ NMR (DMSO- $d_{6}, 400 \mathrm{MHz}, \delta$ ppm): 4.52 (s, 2H, - $\mathrm{Ar}-\mathrm{CH}_{2}-\mathrm{N}-$ ), 5.53 (s, 2H, -O- $\mathrm{CH}_{2}-\mathrm{N}-$ ), 6.86-7.92 (m, 8H, Ar-H); ${ }^{13} \mathrm{C}$ NMR (DMSO- $d_{6}, 75 \mathrm{MHz}, \delta$ ppm): 50.2, 79.3, 106.5, 107.9, 119.5, 124.7, 125.4, 125.9, 126.4, 127.2, 132.6, 133.5, 134.9, 142.6, 147.2, 150.1; MS: $m / z$ 495.1 (m+1); Elemental analysis: $\mathrm{C}_{18} \mathrm{H}_{12} \mathrm{Br}_{3} \mathrm{NOCalcd}$.: C: $43.41 \%$; $\mathrm{H}$ : $2.43 \%$; $\mathrm{N}: 2.81 \%$; Found: C: $43.58 \%$, H: $2.25 \%$, N: $2.64 \%$.

3,4-Dihydro-3-p-tolyl-2H-naphtho[2,1-e][1,3]oxazine (4e): IR (KBr, $v_{\max } / \mathrm{cm}^{-1}$ ): 1021 (sym. C-O-C), 1234 (asym. C-O-C); ${ }^{1} \mathrm{H}$ NMR (DMSO- $\left.d_{6}, 400 \mathrm{MHz}, \delta \mathrm{ppm}\right): 2.40\left(\mathrm{~s}, 3 \mathrm{H}, \mathrm{CH}_{3}\right)$, 4.90 (s, 2H, - Ar- $\left.\mathrm{CH}_{2}-\mathrm{N}-\right), 5.60$ (s, 2H, -O-CH2-N-), 6.60-7.90 (m, 10H, Ar-H); ${ }^{13} \mathrm{C}$ NMR (DMSO- $d_{6}, 75 \mathrm{MHz}, \delta$ ppm): 21.2 , 49.2, 78.8, 110.4, 115.2, 117.8, 119.6, 120.3, 124.5, 125.5, 125.9, 126.3, 127.4, 129.6, 132.1, 147.8, 148.2; MS: $m / z 276.2$ $(\mathrm{m}+1)$; Elemental analysis: $\mathrm{C}_{19} \mathrm{H}_{17} \mathrm{NO}$ Calcd.: C: $82.88 \%$; $\mathrm{H}$ : 6.22\%; N: 5.09\%; Found: C: $82.71 \%$, H: 6.30\%, N: 5.04\%.

3-(2-Ethoxyphenyl)-3,4-dihydro-2H-naphtho[2,1-e][1,3] oxazine (4f): IR ( $\mathrm{KBr}, v_{\max } / \mathrm{cm}^{-1}$ ): 1021 (sym. C-O-C), 1234 (asym. C-O-C); ${ }^{1} \mathrm{H}$ NMR (DMSO- $d_{6}, 400 \mathrm{MHz}, \delta$ ppm): 1.29 (t, $\left.3 \mathrm{H}, J=14 \mathrm{~Hz}, \mathrm{O}-\mathrm{CH}_{2}-\mathrm{CH}_{3}\right), 3.96\left(\mathrm{q}, 2 \mathrm{H}, J=14 \mathrm{~Hz}, \mathrm{O}-\mathrm{CH}_{2}-\right.$ $\left.\mathrm{CH}_{3}\right), 4.61$ (s, 2H, -Ar- $\left.\mathrm{CH}_{2}-\mathrm{N}-\right), 5.40$ (s, 2H, -O-CH $-\mathrm{N}-$ ), 6.17-7.44 (m, 10H, Ar-H); ${ }^{13} \mathrm{C}$ NMR (DMSO- $d_{6}, 75 \mathrm{MHz}, \delta$ ppm): 13.9, 48.8, 64.7, 81.5, 111.8, 114.7, 115.8, 117.5, 118.6, 121.2, 122.2, 124.5, 125.4, 125.7, 126.2, 127.4, 128.8, 133.2, 146.1, 149.3; MS: $m / z$ 306.2 (m+1); Elemental analysis: $\mathrm{C}_{20} \mathrm{H}_{19}$ $\mathrm{NO}_{2}$ Calcd.: C: 78.66\%; H: 6.27\%; N: 4.59\%; Found: C: $78.48 \%$, H: $6.37 \%$, N: $4.67 \%$.

3-(4-Fluorophenyl)-3,4-dihydro-2H-naphtho[2,1-e][1,3]oxazine (4g): IR ( $\mathrm{KBr}, v_{\max } / \mathrm{cm}^{-1}$ ): 1026 (sym. C-O-C), 1245 (asym. C-O-C) ${ }^{1} \mathrm{H}$ NMR (DMSO-d $\left.d_{6}, 400 \mathrm{MHz}, \delta \mathrm{ppm}\right): 4.90$ (s, 2H, $-\mathrm{Ar}-\mathrm{CH}_{2}-\mathrm{N}-$ ), 5.60 (s, 2H, -O-CH $-\mathrm{N}-$ ), 6.80-7.80 (m, 10H, $\mathrm{Ar}-\mathrm{H}) ;{ }^{13} \mathrm{C}$ NMR (DMSO- $\left.d_{6}, 75 \mathrm{MHz}, \delta \mathrm{ppm}\right): 49.2,78.5$, 112.2, 115.7, 116.1, 117.2, 118.2, 122.4, 123.1, 125.1, 125.6, 125.9, 127.5, 128.4, 130.4, 150.2; MS: m/z $280.2(\mathrm{~m}+1)$; Elemental analysis: $\mathrm{C}_{18} \mathrm{H}_{14} \mathrm{FNO}$ Calcd.: C: $77.40 \%$; H: 5.05\%; N: 5.01\%; Found: C: $77.87 \%$, H: 5.14\%, N: 5.15\%.

3,4-Dihydro-3-(3-methoxyphenyl)-2H-naphtho[2,1-e][1,3] oxazine (4h): IR (KBr, $v_{\max } / \mathrm{cm}^{-1}$ ): 1029 (sym. C-O-C), 1211 (asym. C-O-C); ${ }^{1} \mathrm{H}$ NMR (DMSO- $d_{6}, 400 \mathrm{MHz}, \delta$ ppm): 3.54 (s, 3H, OMe), 4.70 (s, 2H, -Ar- $\left.\mathrm{CH}_{2}-\mathrm{N}-\right), 5.50$ (s, 2H, -O- $\mathrm{CH}_{2}-$ N-), 6.21-7.63 (m, 10H, Ar-H); ${ }^{13} \mathrm{C}$ NMR (DMSO- $d_{6}, 75 \mathrm{MHz}$, $\delta$ ppm): 49.1, 53.4, 79.2, 112.1, 114.5, 115.8, 117.3, 118.2, 120.9, 124.5, 125.6, 125.9, 126.5, 127.5, 130.3, 133.1, 146.7, 148.9, 151.1; MS: m/z $292.2(\mathrm{~m}+1)$; Elemental analysis: $\mathrm{C}_{19}$ 
$\mathrm{H}_{17} \mathrm{NO}_{2}$ Calcd.: C: $78.33 \%$; H: $5.88 \%$; N: $4.81 \%$; Found: C: $78.21 \%$, H: $5.78 \%$, N: $4.90 \%$.

3,4-Dihydro-3-o-tolyl-2H-naphtho[2,1-e][1,3]oxazine (4i): IR ( $\mathrm{KBr}, v_{\max } / \mathrm{cm}^{-1}$ ): 1029 (sym. C-O-C), 1231 (asym. C-O-C); ${ }^{1} \mathrm{H}$ NMR (DMSO- $\left.d_{6}, 400 \mathrm{MHz}, \delta \mathrm{ppm}\right): 2.20$ (q, 3H, $\mathrm{CH}_{3}$ ), 4.90 (s, 2H, -Ar-CH $\left.\mathrm{CH}_{2}-\mathrm{N}-\right), 5.70$ (s, 2H, -O-CH $\left.2-\mathrm{N}-\right)$, 6.80-7.90 $(\mathrm{m}, 10 \mathrm{H}, \mathrm{Ar}-\mathrm{H}) ;{ }^{13} \mathrm{C}$ NMR (DMSO- $d_{6}, 75 \mathrm{MHz}, \delta \mathrm{ppm}$ ): 20.2, 50.1, 79.1, 113.2, 116.1, 117.4, 119.2, 120.5, 124.7, 125.8, 126.1, 126.8, 127.9, 129.7, 130.4, 147.2, 148.1, 149.2, 150.4; MS: $m / z$ 276.2 $(\mathrm{m}+1)$; Elemental analysis: $\mathrm{C}_{19} \mathrm{H}_{17} \mathrm{NO}$ Calcd.: C: $82.88 \%$; H: $6.22 \%$; N: $5.09 \%$; Found: C: $82.84 \%, \mathrm{H}$ : $6.68 \%, \mathrm{~N}: 5.14 \%$.

Antimicrobial activity assays. The compounds were diluted in dimethylformamide (DMF) with required concentrations for bioassay. Antimicrobial activity was evaluated by screening of the compounds by standard method i.e. Agar cup plate method against a panel of human pathogenic microorganisms: one Gram positive (Bacillus subtilis NCIM 2250), one Gram negative (Escherichia coli ATCC 25922) were used for the antibacterial assay, while for the antifungal assay, (Candida albicans MTCC 277) and (Aspergillus niger NCIM 545) were used for the studies. Microorganisms were maintained at $37^{\circ} \mathrm{C}$ on Mueller Hinton (MH) agar slants. Moreover the $\mathrm{MH}$ agar and Czapek Dox broth were used to evaluate antibacterial and antifungal activity respectively. To make a judgment of antibacterial and antifungal potency of the synthesized compounds (4a-i) commercial antibiotics such as Streptomycin (Strept.) and Griseofluvin (Gris.) in DMF served as reference standards to compare inhibition of growth. The plate containing bacterial organism were incubated at $37 \pm 0.5^{\circ} \mathrm{C}$ and plates containing fungal organism were incubated at $28 \pm 0.5^{\circ} \mathrm{C}$ for $48 \mathrm{~h}$. The zone of inhibition was calculated by measuring the diameter of zone of inhibition for bacterial and fungal growth around the disc. Averages of three independent determinations were recorded. The minimum inhibitory concentration (MIC) of the samples by Cup plate method on $\mathrm{MH}$ agar plates containing the following concentrations $(\mu \mathrm{g} / \mathrm{mL})$ : 0 (control), 1, 2, 3, 5, 10, 15, 20, 30 and 40. MH was molted and poured in Petri dishes according to National Committee for Clinical Laboratory Standards (NCCLS, M7-A5 January 2000). The plates were incubated at $37{ }^{\circ} \mathrm{C}$, examined after $24 \mathrm{~h}$ and incubated further for $72 \mathrm{~h}$, where necessary. The MIC was defined as the lowest concentration resulting in inhibition of visible bacterial and fungal growth. The MIC determination was performed in triplicate for each organism and the experiment was repeated wherever necessary.

Acknowledgments. The authors would like to thank the Head, Department of Chemistry, Dr. Babasaheb Ambedkar Marathwada University, Aurangabad for constant encouragement and providing necessary facilities.

\section{References}

1. Reichert, B. In Die Mannich Reaction; Springer-Verlag: Berlin, 1959.

2. Blicke, F. F. In The Mannich Reaction: Org reactions; John Wiley: New York, 1942; Vol. 1, pp 303-341.

3. (a) Kuehne, M. E.; Konopke, E. A. J. Med. Chem. 1962, 5, 257. (b) Chylinska, J. B.; Urbanski, T. J. Med. Chem. 1963, 6, 484. (c) Hsu, L. Y.; Lin, C. H. Heterocycles 1996, 43, 2687.

4. (a) Chylinska, J. B.; Janowiec, M.; Urbanski, T. Br. J. Pharmacol. 1971, 43, 649. (b) Latif, N.; Mishriky, N.; Massad, F. Aust. J. Chem. 1982, 35, 1037.

5. (a) Pedersen, O. S.; Pedersen, E. B. Synthesis 2000, 479. (b) Cocuzza, A. J.; Chidester, D. R.; Cordova, B. C.; Jeffrey, S.; Parsons, R. L.; Bacheler, L. T.; Erickson-Viitanen, S.; Trainor, G. L.; Ko, S. S. Bioorg. Med. Chem. Lett. 2001, 11, 1177.

6. Duffin, W. M.; Rollo, I. M. Br. J. Pharmacol. 1957, 12, 171.

7. Singh, O. V.; Han, H. Tetrahedron Lett. 2007, 48, 2345.

8. Joyce, J. N.; Presgraves, S.; Renish, L.; Borwege, S.; Osredkar, D. H.; Replogle, M.; PazSoldan, M.; Millan, M. J. Exp. Neurol. 2003, 184, 393.

9. Kerdesky, F. A. J. Tetrahedron Lett. 2005, 46, 1711.

10. Toure, B. B.; Hall, D. G. Chem. Rev. 2009, 109, 4439.

11. Bondock, S.; Fadaly, W.; Metwally, M. A. J. Sulfur Chem. 2009, 30,74 .

12. Ganem, B. Acc. Chem. Res. 2009, 42, 463.

13. Domling, A. Chem. Rev. 2006, 106, 17.

14. Wu, Y.; He, L. N.; Du, Y.; Wang, J. Q.; Miao, C. X.; Li, W. Tetrahedron 2009, 65, 6204.

15. Zhang, Z. H.; Li, T. S. Curr. Org. Chem. 2009, 13, 1.

16. Das, B.; Krishnaiah, M.; Venkuleswarlu, K. Chem. Lett. 2007, 36,82 .

17. Das, B.; Ravikanth, B.; Reddy, K. R.; Rao, B. V. Helv. Chim. Acta 2007, 90, 105.

18. Sangshetti, J. N.; Kokare, N. D.; Shinde, D. B. Monatshefte fur Chemie. 2007, 138, 1289.

19. Nagawade, R. R.; Shinde, D. B. Russ. J. Org. Chem. 2006, $42,453$.

20. Agag, T. J. App. Poly. Sci. 2006, 100, 3769.

21. Burke, W. J.; Nurdock, K. C. J. Am. Chem. Soc. 1954, 76, 1677.

22. Chernykh, A.; Agag, T.; Ishida, H. Macromolecules 2009, 42, 5121.

23. Bouaziz, Z; Riondel, J.; Mey, A.; Berlionz, M.; Villard, J.; Fillion, H. Eur. J. Med. Chem. 1991, 26, 469.

24. Mathew, B. P.; Nath, M. J. Het. Chem. 2009, 46, 1003.

25. Pokalwar, R. U.; Hangarge, R. V.; Maske, P. V.; Shingare, M. S. Arkivoc 2006, 11, 196.

26. Sapkal, S. B.; Shelke, K. F.; Shingate, B. B.; Shingare, M. S. Tetrahedron Lett. 2009 50, 1754.

27. Sonar, S. S.; Kategaonkar, A. H.; Gill, C. H.; Shingate, B. B.; Shingare, M. S. Arkivoc 2009, 2, 138.

28. Sonar, S. S.; Sadaphal, S. A.; Kategaonkar, A. H.; Pokalwar, R. U.; Shingate, B. B. Shingare, M. S. Bull. Korean Chem. Soc. 2009, 30,825 .

29. Kategaonkar, A. H.; Pokalwar, R. U.; Sonar, S. S.; Gawali, V. U.; Shingate, B. B.; Shingare, M. S. Eur. J. Med. Chem. 2010, 45, 1128 .

30. Kategaonkar, A. H.; Pokalwar, R. U.; Sadaphal, S. A.; Shinde, P. V.; Shingate, B. B.; Shingare, M. S. Heteroatom Chem. 2009, 20 , 436. 